\title{
Transmission Switching in Joint Energy and Spinning Reserve Markets Considering Stability Criteria
}

\author{
Rahmat Aazami $^{1,2}$, Farzad Soltanian² ${ }^{2}$ Masoud Moradkhani ${ }^{2}$, and Javad Rashidbeigi ${ }^{2}$ \\ ${ }^{1}$ Faculty of Engineering, Ilam University, Ilam, Iran. \\ ${ }^{2}$ Department of Electrical Engineering, Ilam Branch, Islamic Azad University, Ilam, Iran. \\ azami.rahmat@yahoo.com
}

\begin{abstract}
There is a great resolution calling for smart grids in recent years. Introduction of new technologies, that make the network flexible and controllable, is a main part of smart grid concept and a key factor to its success. Transmission network as a part of system network has drawn less attention. Transmission switching as a transmission service can release us from load shedding and remove the constraints' violations.

In addition to removing the congestion and decreasing the system cost, transmission switching may damage generating units due to transient states in instance of reconfiguration. Therefore, in optimal transmission switching, the system security, practical limitations and possible damages should be considered.

In this paper transmission switching is modeled in probabilistic energy and spinning reserve day-ahead market clearing problem to guaranty the network security standards.

The results show that proposed transmission switching in addition to significant decrease in system cost, can improve the other network characteristics such as system reliability.
\end{abstract}

Keywords: smart grids, transmission switching, security, day-ahead market

\section{Introduction}

Transmission switching studies were of the interest to the researches from Eighties. In primary studies, the main focus was decreasing the load shedding. Next, the efficiency of optimal transmission switching to solve other operation issues such as voltage drop, network loss and system security was analyzed. After restructuring in power systems and introduction of smart grid concept, transmission switching problem was redefined in the new environment [1-7]. The switching problem can be analyzed from two standpoints.

Corrective switching: From the first standpoint, the switching is applied as a smart facility in emergency states such as fault conditions. In this situation transmission system topology is selected in post fault conditions in a way that network constraints are satisfied.

Preventive switching: From the second standpoint, the main purpose of switching is system cost reduction. In the case of congestion, some cheaper generating units cannot produce their maximum output. This increases the system cost. In this situation the switching should remove the congestion and reduce the production cost.

Switching was used in [8-9] for congestion removal. A method based on DC Optimal Power Flow (OPF) was used in these references. In [10-11] the N-1 security criteria have been added to the model presented in [8-9].

In [12-14], heuristic methods were used to restrict the search space and therefore, to reduce the solution time. In these papers the lines with highest impact on congested lines were categorized based on a sensitivity analysis. For each category as a candidate of switching, the system cost was calculated and finally the most economical option was taken. It should be noted that since unit generations and switching decisions are found separately, the solution may be suboptimal.

Received: October $9^{\text {th }}, 2014$. Accepted: November $4^{\text {th }}, 2014$ 
In most of studies on transmission switching only the DC network constraints have been considered and the AC constraints voltage security constraints and reactive load flow have been neglected. Since the switching may cause violation in voltage constraints as well as other AC constraints, the methods presented based on DC load flow are less efficient [15].

On the other hand the AC constraints cause nonlinearity in the problem. Therefore, with these constraints the switching problem is a Mixed Integer Non-linear Programming (MINLP) problem. These problems take so long to be solved and it is possible that no solution is found. The global optimality is also not guaranteed. Problem decomposition has been proposed to solve the issue.

Reference [15] found the switching scheme and generation schedule using a DC OPF at the first step. The results then were tested using an AC power flow and in the case of constraint violation, the switching scheme was ban and a new switching scheme was found. As the result of separation of DC and AC sub-problems this method also fails to guarantee the global optimum solution.

In [15-17] Benders decomposition was used. In the main problem, the generation schedule and switching scheme was found based on DC OPF. In sub-problems, AC constraints were checked and in the case of violation the violated constraints were linked to the main problem. These newly introduced constraints change the results of main problem to remove the constraint violations in sub-problem.

Security constraints were included in [17] through N-1 criteria. The security checking subproblem was not linked to the main problem. This restricts the chance of global optimum solution. The method presented to find the order of switching has also some problems that cause the solution to deviate from the optimum solution in some cases.

On the other hand, though the switching can be useful in transmission networks, it may damage the generating units in some instances. The main reason is introducing a relatively high torques on the rotor of these units in reconfigurations. In this paper the transient stability criterion is included in the model presented for energy and spinning reserve clearing to restrict the chance of damages.

Based on the results of researches that some of them have been reported in this section, transmission switching can be useful for operation cost reduction. However, this switching may cause the system instability in some instances. This increase the network security cost. This paper analyses and models the transmission switching with dynamic constraints in a probabilistic co-optimization model for energy and spinning reserve scheduling. Using this model the safe operation considering the dynamic switching constraints has been guaranteed.

\section{Modeling of Transmission Switching and Dynamic Constraints in Probabilistic Co- optimization of Energy and Spinning Reserve Markets}

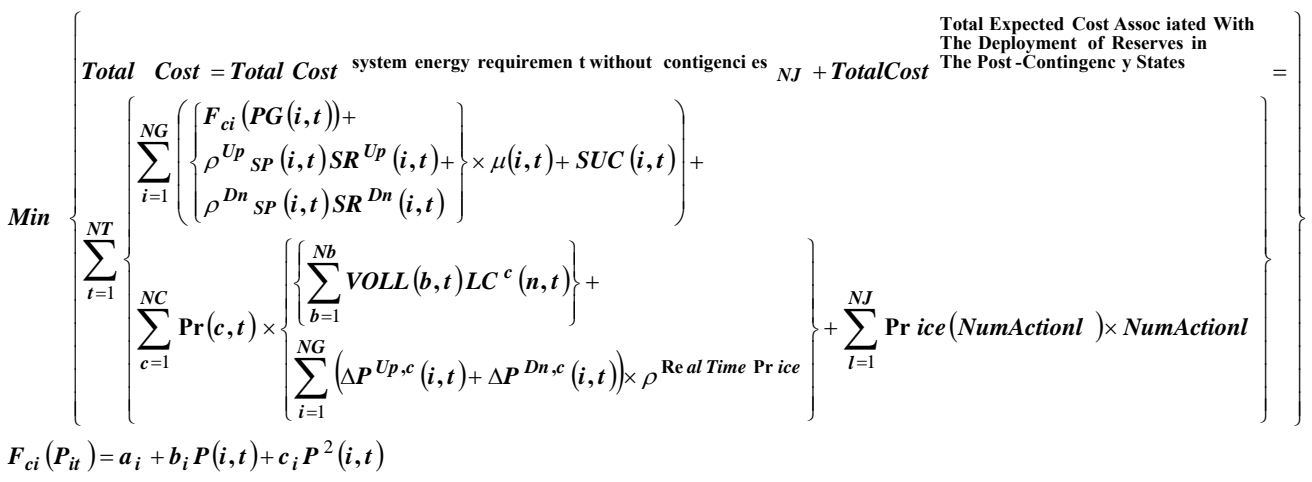


The cost of switching, including the cost of opening and closing operations and the cost related to the depreciation of the switch insulators, is modeled based on the reinvestment costs for installing the new switches. In a short period, the cost of switching is considered to be proportional to the number of switching operations.

In [18-30] different MINLP problem were solved in different engineering branches including the Unit Commitment (UC) problem. In this section a probabilistic MINLP model is proposed for co-optimization of day-ahead energy and reserve markets. Switching capability is just considered for some network lines. The objective function of (1) is considered to minimize the energy, spinning reserve and switching costs in a 24 hour time period.

In (1), units' production costs, startup costs and reserve capacity costs are shown in the first term. The second term includes load shedding costs and the cost associate with reserve applications (change in production schedule). Switching cost is shown in third term.

The network and units' constraints should be considered for both pre- and post-contingency states. The constraints can be divided into two groups, post-contingency and pre-contingency constraints.

\section{A. Pre-contingency constraints}

All the variables are considered to be positive. Minimum and maximum producible power constraints considering the spinning reserves are given in (2) for different units in different hours.

$$
\begin{aligned}
& \left(P^{\min }(i)+S_{R^{D n}}(i, t)\right) \times \mu(i, t) \leq P(i, t) \leq\left(P^{\max }(i)-S R^{U p}(i, t)\right) \times \mu(i, t) \\
& i=1,2, \ldots N G \quad t=1, \ldots N T
\end{aligned}
$$

Reactive power minimum and maximum limits of the units are give

$$
Q^{\min }(i, t) \leq Q G(i, t) \leq Q^{\max }(i, t)
$$

Startup cost is modeled in (4).

$$
\boldsymbol{S U C}(\boldsymbol{i}, \boldsymbol{t}) \geq(\mu(\boldsymbol{i}, \boldsymbol{t})-\mu(\mathbf{i}, \boldsymbol{t}-1)) \times \boldsymbol{S U C}(\mathbf{i}) \quad \boldsymbol{i}=1,2, \ldots N G \quad t=1, \ldots N \boldsymbol{N}
$$

Ramping constraints are given in (5).

$$
\begin{aligned}
& \boldsymbol{P}(\boldsymbol{i}, \boldsymbol{t})-\boldsymbol{P}(\boldsymbol{i}, \boldsymbol{t}-1) \leq[1-\mu(\boldsymbol{i}, \boldsymbol{t})(1-\mu(\boldsymbol{i}, \boldsymbol{t}-1))] \times \boldsymbol{R} \boldsymbol{R} \boldsymbol{U}(\boldsymbol{i}) \quad \boldsymbol{i}=1, \ldots, \boldsymbol{N} \boldsymbol{G} \\
& +\mu(\mathbf{i}, \boldsymbol{t}) \times(1-\mu(\mathbf{i}, \boldsymbol{t}-1)) \times \boldsymbol{P}^{\min }(\mathbf{i}) \\
& \boldsymbol{P}(\boldsymbol{i}, \boldsymbol{t}-1)-\boldsymbol{P}(\boldsymbol{i}, \boldsymbol{t}) \leq[1-\mu(\boldsymbol{i}, \boldsymbol{t}-1)(1-\mu(\boldsymbol{i}, \boldsymbol{t}))] \times \boldsymbol{R R D}(\boldsymbol{i}) \quad \boldsymbol{i}=1, \ldots, \boldsymbol{N G} \\
& +\mu(\boldsymbol{i}, \boldsymbol{t}-1) \times(1-\mu(\boldsymbol{i}, \boldsymbol{t})) \times \boldsymbol{P}^{\min }(\boldsymbol{i})
\end{aligned}
$$

Minimum up- and down-time constraints are given in (6).

$$
\begin{aligned}
& {\left[\boldsymbol{X}_{\boldsymbol{i}(\boldsymbol{t}-1)}^{\text {on }}-\boldsymbol{T}_{\boldsymbol{i}}^{\text {on }}\right] \times[\mu(\boldsymbol{i}, \boldsymbol{t}-1)-\mu(\boldsymbol{i}, \boldsymbol{t})] \geq 0 \quad \boldsymbol{i}=1, \ldots, \boldsymbol{N G} \quad \boldsymbol{t}=1, \ldots, \boldsymbol{N T}} \\
& {\left[\boldsymbol{X}_{\boldsymbol{i}(\boldsymbol{t}-1)}^{\text {off }}-\boldsymbol{T}_{\boldsymbol{i}}^{\text {off }}\right] \times[\mu(\boldsymbol{i}, \boldsymbol{t})-\mu(\mathbf{i}, \boldsymbol{t}-1)] \geq 0}
\end{aligned}
$$

The maximum sustainable ramp rate limits the reserve that can be applied in 10 minutes. These constraints are given in (7), where $M S R R$ is the maximum sustainable ramp rate in $\mathrm{MW} / \mathrm{min}$.

$$
\begin{aligned}
& 0 \leq \mathrm{SR}^{\mathrm{UP}}(\mathrm{i}, \mathrm{t}) \leq \mathrm{MSRRU}(\mathrm{i}) \mathrm{x} 10 \mathrm{~min} \\
& 0 \leq \mathrm{SR}^{\mathrm{DN}}(\mathrm{i}, \mathrm{t}) \leq \operatorname{MSRRD}(\mathrm{i}) \times 10 \mathrm{~min} \mathrm{i}=1,2, \ldots \mathrm{NG} \mathrm{t}=1, \ldots \mathrm{NT}
\end{aligned}
$$


Rahmat Aazami, et al.

Equation (8) gives the pre-contingency load balance constraint

$$
\sum_{i=1}^{N G} P G(i, t)=\sum_{b=1}^{N b} P L(b, t)
$$

The net injected active and reactive power to each bus should be respectively equal to the active and reactive power output via transmission lines connected to this bus. These constraints are given in (9).

$$
\begin{aligned}
& \sum_{i=1}^{N G b} P G(i, t)-P L(b, t)=Z(l, t) \times \sum_{l=1}^{L b} V_{b}^{2}\left(G_{l}+0.5 G_{l 0}\right)-V_{b} V_{m}\left(\begin{array}{l}
G_{l} \cos (\delta(n, t)-\delta(m, t))+ \\
B_{l} \sin (\delta(n, t)-\delta(m, t))
\end{array}\right) \\
& \sum_{i=1}^{N G b} Q G(i, t)-Q L(b, t)=Z(l, t) \times \sum_{l=1}^{L b}-V_{b}^{2}\left(B_{l}+0.5 B_{l 0}\right)-V_{b} V_{m}\left(\begin{array}{l}
G_{l} \sin (\delta(n, t)-\delta(m, t))- \\
B_{l} \cos (\delta(n, t)-\delta(m, t))
\end{array}\right) \\
& \quad b=1,2, . ., N b
\end{aligned}
$$

The line active and reactive flow limits are given in (10), considering the switchable lines.

$$
\begin{aligned}
& \left\{\begin{array}{l}
\operatorname{Re}\left\{V_{n} e^{j \delta_{n}(I, t)}\left(\begin{array}{l}
0.5 \times\left(G_{I 0}+j B_{k 0}\right) V_{n} e^{j \delta_{n}(I, t)}+ \\
\left(G_{I}+j B_{k}\right) \times\left(V_{n} e^{j \delta_{n}(I, t)}-V_{m} e^{j \delta_{m}(I, t)}\right)
\end{array}\right)^{*}\right\}-P(I, t)+(1-Z(I, t)) \times M \geq 0 \\
\operatorname{Re}\left\{V_{n} e^{j \delta_{n}(I, t)}\left(\begin{array}{l}
0.5 \times\left(G_{I 0}+j B_{k 0}\right) V_{n} e^{j \delta_{n}(I, t)}+ \\
\left(G_{I}+j B_{k}\right) \times\left(V_{n} e^{j \delta_{n}(I, t)}-V_{m} e^{j \delta_{m}(I, t)}\right)
\end{array}\right)^{*}\right\}-P(I, t)-(1-Z(I, t)) \times M \leq 0
\end{array}\right. \\
& \operatorname{Im}\left\{V_{n} e^{j \delta_{n}(I, t)}\left(\begin{array}{l}
0.5 \times\left(G_{I 0}+j B_{k 0}\right) V_{n} e^{j \delta_{n}(l, t)}+ \\
\left(G_{I}+j B_{k}\right) \times\left(V_{n} e^{j \delta_{n}(l, t)}-V_{m} e^{j \delta_{m}(I, t)}\right)
\end{array}\right)^{*}\right\}-Q(I, t)+(1-Z(I, t)) \times M \geq 0 \\
& \left\{\operatorname{Im}\left\{V_{n} e^{j \delta_{n}(I, t)}\left(\begin{array}{l}
0.5 \times\left(G_{I 0}+j B_{k 0}\right) V_{n} e^{j \delta_{n}(l, t)}+ \\
\left(G_{I}+j B_{k}\right) \times\left(V_{n} e^{j \delta_{n}(l, t)}-V_{m} e^{j \delta_{m}(I, t)}\right)
\end{array}\right)\right\}-Q(I, t)-(1-Z(I, t)) \times M \leq 0\right. \\
& I=1,2, . .9 N J, \ldots, N I \quad t=1, \ldots N T
\end{aligned}
$$

Apparent power flow of the lines cannot be higher than the line flow limits. This constraint is shown in (11).

$$
\begin{aligned}
& -Z(l, t) \times S^{\max }(l, t) \leq S(l, t) \leq Z(l, t) \times S^{\max }(l, t) \\
& S(l, t)=\sqrt{Q(l, t)^{2}+P(l, t)^{2}} \quad l=1, \ldots, N J, \ldots, N L \quad t=1, \ldots, N T
\end{aligned}
$$

The number of switching operation cannot be higher than maximum allowable switching numbers.

$$
\text { NumActionl }=\sum_{t-1}^{24}\{\mid Z(l, t)-Z(l, t-1)\} \leq \text { NumAction }^{\max }
$$

\section{B. Post Contingency Constraints}

The change in generation of each unit is lower than the regarding committed reserve (13).

$$
\begin{aligned}
& 0 \leq \Delta P^{U p}(i, t) \leq S R^{U p}(i, t) \xi^{c}(i, t) \\
& 0 \leq \Delta P^{D n}(i, t) \leq S R^{D n}(i, t) \xi^{c}(i, t)
\end{aligned}
$$


The load shedding constraints for all credible contingencies can be expressed as those of (14).

$$
\begin{aligned}
& \sum_{b=1}^{N b} L C^{c}(b, t)=W \times\left\{\sum_{i=1}^{N G} P(i, t)\left(1-\xi^{c}(i, t)\right)+\sum_{i=1}^{N G} S R^{U p}(i, t)\left(1-\xi^{c}(i, t)\right)-\sum_{i=1}^{N G} S R^{D n}(i, t)\left(\xi^{c}(i, t)\right)\right\} \\
& \frac{\left\{\sum_{i=1}^{N G} P(i, t)\left(1-\xi^{c}(i, t)\right)+\sum_{i=1}^{N G} S R^{U p}(i, t)\left(1-\xi^{c}(i, t)\right)-\sum_{i=1}^{N G} S R^{D n}(i, t)\left(\xi^{c}(i, t)\right)\right\}}{\sum_{i=1}^{N G} P^{\max }(i, t)} \leq W \leq 1+ \\
& \left\{\sum_{i=1}^{N G} P(i, t)\left(1-\xi^{c}(i, t)\right)+\sum_{i=1}^{N G} S R^{U p}(i, t)\left(1-\xi^{c}(i, t)\right)-\sum_{i=1}^{N G} S R^{D n}(i, t)\left(\xi^{c}(i, t)\right)\right\} \\
& \sum_{i=1}^{N G} P^{\max }(i, t) \\
& 0 \leq \boldsymbol{L} \boldsymbol{C}^{\mathrm{c}}(\boldsymbol{b}, \boldsymbol{t}) \leq \boldsymbol{P} \mathbf{L}(\boldsymbol{b}, \boldsymbol{t}) \\
& t=1,2, \ldots N T \quad c=1, \ldots N C
\end{aligned}
$$

Equation (15) shows the post-contingency load balance constraints.

$$
\begin{aligned}
& \sum_{i=1}^{N G b} \xi^{c}(i, t) \times P G(i, t)+\sum_{i=1}^{N G b} \xi^{c}(i, t) \times S R^{U p}(i, t)-\sum_{i=1}^{N G b} \xi^{c}(i, t) \times S R^{D n}(i, t)+L C^{c}(b, t)-P L(b, t)= \\
& \sum_{l=1}^{L b} \mu^{c}(\boldsymbol{l}, \boldsymbol{t}) \times \mathbf{Z}(\boldsymbol{l}, \boldsymbol{t}) \times V_{\boldsymbol{b}}^{2}\left(\boldsymbol{G}_{\boldsymbol{I}}+0.5 \boldsymbol{G}_{I 0}\right)-\boldsymbol{V}_{\boldsymbol{b}} \boldsymbol{V}_{\boldsymbol{m}}\left(\begin{array}{l}
\boldsymbol{G}_{\boldsymbol{l}} \cos (\delta(\boldsymbol{n}, \boldsymbol{t})-\delta(\boldsymbol{m}, \boldsymbol{t}))+ \\
\boldsymbol{B}_{\boldsymbol{I}} \sin (\delta(\boldsymbol{n}, \boldsymbol{t})-\delta(\boldsymbol{m}, \boldsymbol{t}))
\end{array}\right) \\
& \sum_{i=1}^{N G b} \xi^{c}(\boldsymbol{i}, t) Q G(i, t)-Q L(b, t)=\mu^{c}(\boldsymbol{l}, \boldsymbol{t}) \times \mathbf{Z}(\boldsymbol{l}, \boldsymbol{t}) \times \sum_{l=1}^{L b}-V_{b}^{2}\left(B_{I}+0.5 B_{I 0}\right)- \\
& \boldsymbol{V}_{\boldsymbol{b}} \boldsymbol{V}_{\boldsymbol{m}}\left(\begin{array}{l}
\boldsymbol{G}_{\boldsymbol{l}} \sin (\delta(\boldsymbol{n}, \boldsymbol{t})-\delta(\boldsymbol{m}, \boldsymbol{t}))- \\
\boldsymbol{B}_{\boldsymbol{l}} \cos (\delta(\boldsymbol{n}, \boldsymbol{t})-\delta(\boldsymbol{m}, \boldsymbol{t}))
\end{array}\right) \quad \boldsymbol{b}=1,2, \ldots, \boldsymbol{N b} \quad \boldsymbol{c}=1,2, \ldots, \boldsymbol{N C}
\end{aligned}
$$

The post-contingency line active and reactive limits, considering the switchable lines, are shown in (16).

$$
\begin{aligned}
& \int \operatorname{Re}\left\{V_{\boldsymbol{n}} \boldsymbol{e}^{j \delta_{n}(I, t)}\left(\begin{array}{l}
0.5 \times\left(G_{I 0}+j B_{k 0}\right) V_{n} e^{j \delta_{n}(I, t)}+ \\
\left(G_{I}+j B_{k}\right) \times\left(V_{n} e^{j \delta_{n}(I, t)}-V_{m} e^{j \delta_{m}(l, t)}\right)
\end{array}\right)^{*}\right\}-P^{c}(I, t)+\mu^{c}(I, t) \times(1-Z(I, t)) \times M \geq 0 \\
& \operatorname{Re}\left\{V_{n} e^{j \delta_{n}(I, t)}\left(\begin{array}{l}
0.5 \times\left(G_{l 0}+j B_{k 0}\right) V_{n} e^{j \delta_{n}(I, t)}+ \\
\left(G_{l}+j B_{k}\right) \times\left(V_{n} e^{j \delta_{n}(I, t)}-V_{m} e^{j \delta_{m}(I, t)}\right)
\end{array}\right)\right\}-P^{c}(I, t)-\mu^{c}(I, t) \times(1-Z(I, t)) \times M \leq 0 \\
& \left.\int \operatorname{Im}\left\{V_{n} e^{j \delta_{n}(l, t)}\left(\begin{array}{l}
0.5 \times\left(G_{I 0}+j B_{k 0}\right) V_{n} e^{j \delta_{n}(I, t)}+ \\
\left(G_{l}+j B_{k}\right) \times\left(V_{n} e^{j \delta_{n}(I, t)}-V_{m} e^{\left.j \delta_{m}(I, t)\right)}\right.
\end{array}\right)\right)^{*}\right\}-Q^{c}(I, t)+\mu^{c}(I, t) \times(1-Z(I, t)) \times M \geq 0 \\
& \left\{\operatorname{Im}\left\{V_{n} e^{j \delta_{n}(I, t)}\left(\begin{array}{l}
0.5 \times\left(G_{I 0}+j B_{k 0}\right) V_{n} e^{j \delta_{n}(l, t)}+ \\
\left(G_{l}+j B_{k}\right) \times\left(V_{n} e^{j \delta_{n}(l, t)}-V_{m} e^{j \delta_{m}(I, t)}\right)
\end{array}\right)\right\}-Q^{c}(I, t)-\mu^{c}(I, t) \times(1-Z(I, t)) \times M \leq 0\right. \\
& \boldsymbol{l}=1,2, . . . \mathrm{NJ}, \ldots, \mathrm{Nl} \quad t=1, \ldots N T \quad c=1, \ldots, N C
\end{aligned}
$$

Post contingency apparent power flow limit is given in (17).

$$
\begin{aligned}
& -Z(I, t) \times \mu^{c}(I, t) \times S^{\max }(I, t) \leq S^{c}(I, t) \leq Z(I, t) \times \mu^{c}(I, t) \times S^{\max }(l, t) \\
& S^{c}(I, t)=\sqrt{P^{c}(I, t)+Q^{c}(I, t)} \\
& I=1, \ldots, N J, \ldots, N L \quad t=1, \ldots, N T
\end{aligned}
$$


The credible contingencies include line and unit outages. Here, a bi-state model is considered for system lines and units. In a power system including lines and units indexed by $i$, with forced outage rate of $\lambda_{i}$ (18) shows the probability of contingency $c$.

$$
\begin{aligned}
& \operatorname{Pr}(c, t)=\prod_{i \in G_{c}} \operatorname{ORR}(i, t) \times \prod_{l \in L_{c}} \operatorname{ORR}(I, t) \\
& \operatorname{ORR}(\boldsymbol{i}, \boldsymbol{t}) \approx \lambda_{\boldsymbol{i}} \times \boldsymbol{T}
\end{aligned}
$$

In (18) and (19) $\lambda_{i}$ is the forced outage rate of element $i$ and $G_{c}$ and $L_{c}$ are respectively the set of affected units and lines in contingency $c . T$ is the market period which is one hour in this study.

Though transmission switching can be useful in smart grids, it may have some adverse effects on generating units. The main reason is high torques appeared on the rotors as the result of reconfiguration. Therefore, in a safe switching scheme it is necessary to limit the rotor angles. In this paper these limitations are introduced to the model as dynamic constraints. The reduced admittance matrix is used to model the switchable lines in these constraints.

In order to model the dynamic constraints, synchronous machine classical model has been used. In this model the transient stability equations are as follows, considering a constant field voltage.

$$
\begin{aligned}
& \dot{\gamma}(\mathbf{i})=\Omega_{\boldsymbol{b}} \times(\boldsymbol{w}(\mathbf{i})-1) \\
& \dot{\boldsymbol{w}}(\mathbf{i})=\frac{1}{\boldsymbol{M}(\boldsymbol{i})} \times\left(\boldsymbol{P}_{\boldsymbol{G}}(\mathbf{i})-\boldsymbol{P}_{\boldsymbol{e}}(\mathbf{i})\right)
\end{aligned}
$$

In (20), $\mathrm{P}_{\mathrm{G}}(\mathrm{i})$ is the input mechanical power of unit, which is considered to be constant. $\Omega_{b}$ is the rate of frequency and $w(i)$ and $\gamma(i)$ are the rotor speed in per unit and rotor angle of unit $i$ respectively. The unit inertia constant is $M(i)$. the electrical power output of the unit can be written as equation (21).

$$
\boldsymbol{P e}(\mathbf{i}, \boldsymbol{t})=\boldsymbol{E}(\mathbf{i}) \sum_{j} \boldsymbol{E}(\boldsymbol{j})\left[\boldsymbol{B}_{i j}(\boldsymbol{t}) \times \sin (\gamma(\boldsymbol{i}, \boldsymbol{t})-\gamma(\boldsymbol{j}, \boldsymbol{t}))+\boldsymbol{G}_{i j}(\boldsymbol{t}) \times \cos (\gamma(\mathbf{i}, \boldsymbol{t})-\gamma(\boldsymbol{j}, \boldsymbol{t}))\right]
$$

In (21), $E(i)$ is the electrical motive force of stator field. $B_{i j}(t)$ and $G_{i j}(t)$ are the element of row $i$ and column $j$ of reduced susceptance and reduced conductance matrices respectively.

$$
\begin{aligned}
& \gamma^{n+1}(i, t)=\gamma^{n}(i, t)+\frac{\Delta t}{2} \times\left(w^{n+1}(i, t)+w^{n}(i, t)-2\right) \\
& w^{n+1}(i, t)=w^{n}(i, t)+\frac{\Delta t}{2 M(i, t)} \times\left(P G(i, t)-P e^{n}(i, t)+P G(i, t)-P e^{n+1}(i, t)\right) \\
& n=1, \ldots, \text { Nend } \quad i=1, \ldots, N G \\
& \operatorname{Pe}^{n}(i, t)=E(i) \sum_{j} E(j)\left[B_{i j}^{n}(t) \times \sin \left(\gamma^{n}(i, t)-\gamma^{n}(j, t)\right)+G_{i j}^{n}(t) \times \cos \left(\gamma^{n}(i, t)-\gamma^{n}(j, t)\right)\right] \\
& Y_{\text {bus }}=G_{\text {bus }}+j B_{\text {bus }}=\left[\begin{array}{cccc}
y_{11} & y_{12} \times Z^{n}(l, t) & \cdots & y_{1 n} \\
y_{12} \times Z^{n}(I, t) & \vdots & \vdots & \vdots \\
\vdots & \vdots & \vdots & \vdots \\
y_{n 1} & \cdots & \cdots & y_{n n}
\end{array}\right] \\
& \mathbf{Z}^{1}(\boldsymbol{I}, \boldsymbol{t})=\mathbf{Z}(\boldsymbol{l}, \boldsymbol{t}-1) \\
& Z^{n}(l, t)=Z^{n+1}(l, t)=Z(l, t) \quad n=2, \ldots, \text { Nend }-1 \\
& G_{\text {loadi }}=P_{\text {loadi }} / V_{i}^{2}, B_{\text {loadi }}=Q_{\text {loadi }} / V_{i}^{2} i=1, \ldots . . N b
\end{aligned}
$$


The rotor angle and speed can be found through dividing the time span of transient state into Nend steps using (22). In these equations $\Delta t$ is the length of time steps and Nend is the number of time steps indexed by $n$. Considering the switchable lines $\mathrm{Bn}(\mathrm{t})$ and $\mathrm{Gn}(\mathrm{t})$ can be defined using (24).

Finally the safe switching constraint is given in (25) for unit $i$.

$$
\gamma^{n}(\boldsymbol{i}, \boldsymbol{t})-\frac{\sum_{k=1}^{N G} \boldsymbol{H}_{\boldsymbol{k}} \gamma^{\boldsymbol{n}}(\boldsymbol{k}, \boldsymbol{t})}{\sum_{\boldsymbol{k}=1}^{N G} \boldsymbol{H}_{\boldsymbol{k}}} \leq \gamma^{\text {critical }}(\boldsymbol{i}, \boldsymbol{t})
$$

The formulation presented in (1) to (25) models the probabilistic joint energy and reserve problem considering the transmission switching. Numerical results are provided in next section.

\section{Numerical Results}

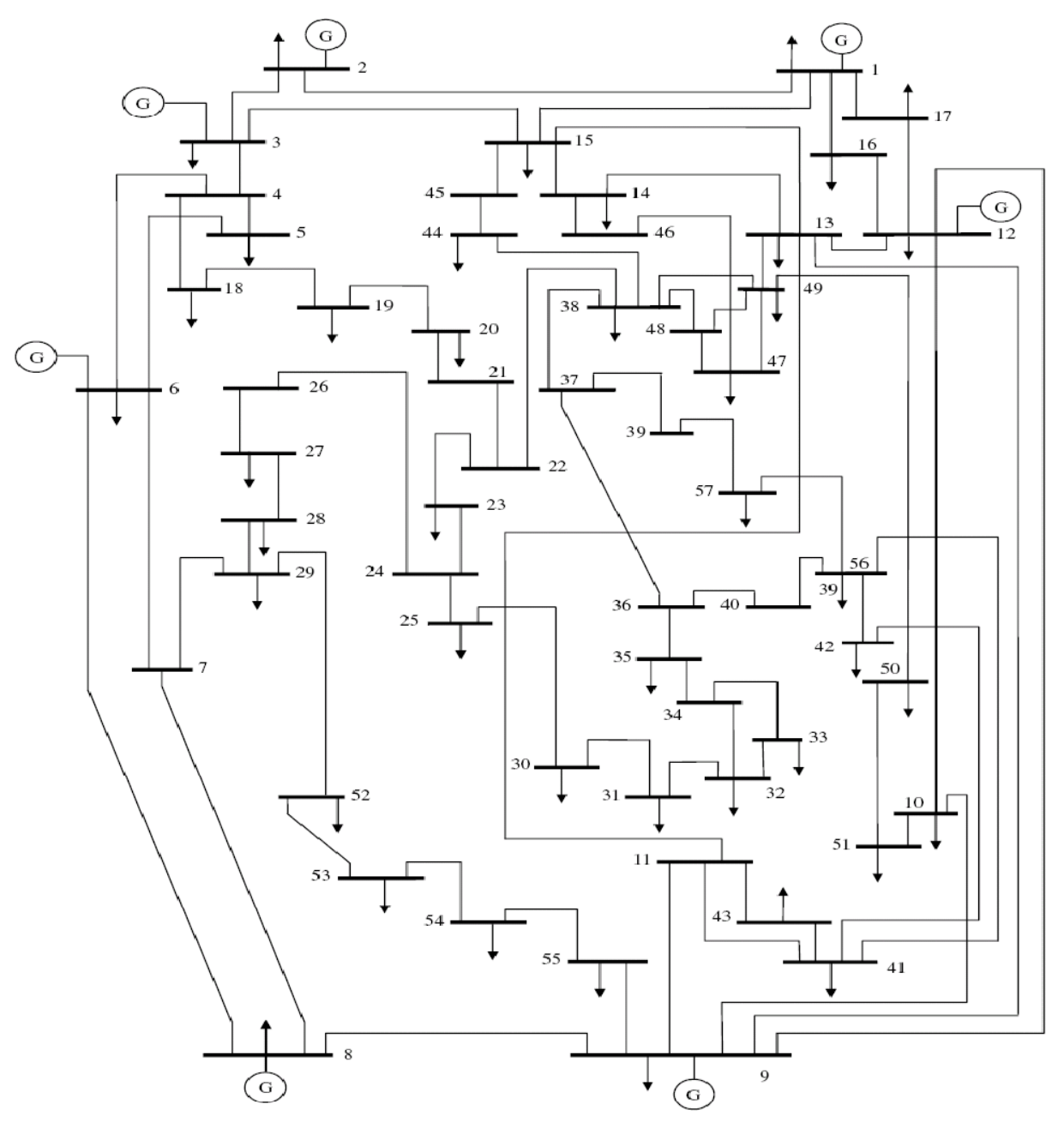

Figure 1. Single phase diagram of 57-bus system 
IEEE 57 bus system is selected to analyze the results of considering the transmission switching in a probabilistic joint energy and reserve problem. This system contains 80 lines and 7 generation units. The total load is $1250.8 \mathrm{MW}$. The other data including the units' offers and electrical characteristics can be found in [31]. The single phase diagram is given in figure 1.

Candidate lines for switching operation are:

$\{1-2,12-16,3-4,13-15,44-48,48-49,22-38,8-9,7-29$ and 1-15

To analyze the effect of switching operations the following case studies will be analyzed:

a) Joint energy and reserve clearing without switching.

b) Joint energy and reserve clearing with switching neglecting the transient stability constraints.

c) Joint energy and reserve clearing with switching considering the transient stability constraints.

Tables 1 and 2 show the UC status in joint energy and reserve clearing without switching operation. Energy and reserve costs are 319156 \$ 59840 \$ respectively for this case. The probabilistic security cost (cost of applied reserve) is $62544 \$$ in this case. The average marginal prices are given in figure 2 for different hours.

Table 1. UC status in energy market

\begin{tabular}{|c|c|c|c|c|c|c|c|c|c|c|c|c|c|c|c|c|c|c|c|c|c|c|c|c|}
\hline \multicolumn{24}{|c|}{24 hours time } & \multirow{2}{*}{$\begin{array}{c}\begin{array}{c}\text { Bus } \\
\text { Generator }\end{array} \\
1\end{array}$} \\
\hline 1 & 1 & 1 & 1 & 1 & 1 & 1 & 1 & 1 & 1 & 1 & 1 & 1 & 1 & 1 & 1 & 1 & 1 & 1 & 1 & 1 & 1 & 1 & 1 & \\
\hline 1 & 1 & 1 & 1 & 1 & 1 & 1 & 1 & 1 & 1 & 1 & 1 & 1 & 1 & 1 & 1 & 1 & 1 & 1 & 1 & 1 & 1 & 1 & 1 & 2 \\
\hline 1 & 1 & 1 & 1 & 1 & 1 & 1 & 1 & 1 & 1 & 1 & 1 & 1 & 1 & 1 & 1 & 1 & 1 & 1 & 1 & 1 & 1 & 1 & 1 & 3 \\
\hline 0 & 0 & 1 & 1 & 1 & 1 & 1 & 1 & 1 & 1 & 1 & 1 & 1 & 1 & 1 & 1 & 0 & 0 & 0 & 0 & 0 & 0 & 0 & 0 & 6 \\
\hline 0 & 0 & 1 & 1 & 1 & 1 & 1 & 1 & 1 & 1 & 1 & 1 & 1 & 1 & 1 & 0 & 0 & 0 & 0 & 0 & 0 & 0 & 0 & 0 & 8 \\
\hline 0 & 0 & 1 & 1 & 1 & 1 & 1 & 1 & 1 & 1 & 1 & 0 & 0 & 0 & 0 & 0 & 0 & 0 & 0 & 0 & 0 & 0 & 0 & 0 & 9 \\
\hline 1 & 1 & 1 & 1 & 1 & 1 & 1 & 1 & 1 & 1 & 1 & 1 & 1 & 1 & 1 & 1 & 1 & 1 & 1 & 1 & 1 & 1 & 1 & 1 & 12 \\
\hline
\end{tabular}

Table 2- UC status in spinning reserve market

\begin{tabular}{|l|l|l|l|l|l|l|l|l|l|l|l|l|l|l|l|l|l|l|l|l|l|l|l|l|}
\hline \multicolumn{10}{|c|}{ B } & \multicolumn{10}{c|}{ Bus } \\
Generator \\
\hline 1
\end{tabular}

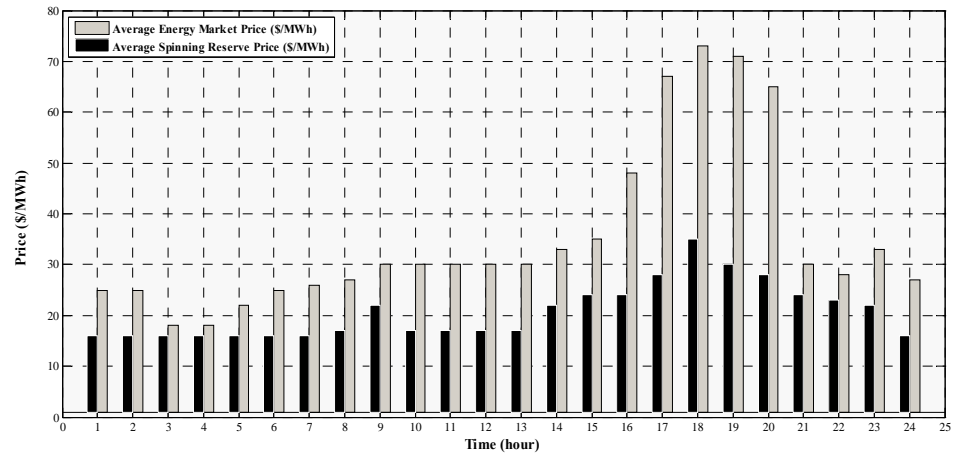

Figure 2. Average marginal price in energy and reserve markets 
- Joint energy and reserve clearing with switching operations neglecting the transient stability constraints

The dynamic constraints are neglected in this case. The UC status is given in Tables 3 and 4 for energy and reserve markets respectively. Comparing to the previous study, the status of some units has not been changed. However, production of the expensive units has been decreased. The switching order is given in Table 5. Table 6 compares the costs of case (a) to those associated with this case. As can be seen the energy, reserve and security cost are reduced by $8 \%, 7 \%$ and $4 \%$ of the values reported in case (a). For switching operations the switching status of hour 0 is considered to be same as the base case.

Table 3. UC status in energy market, case (b)

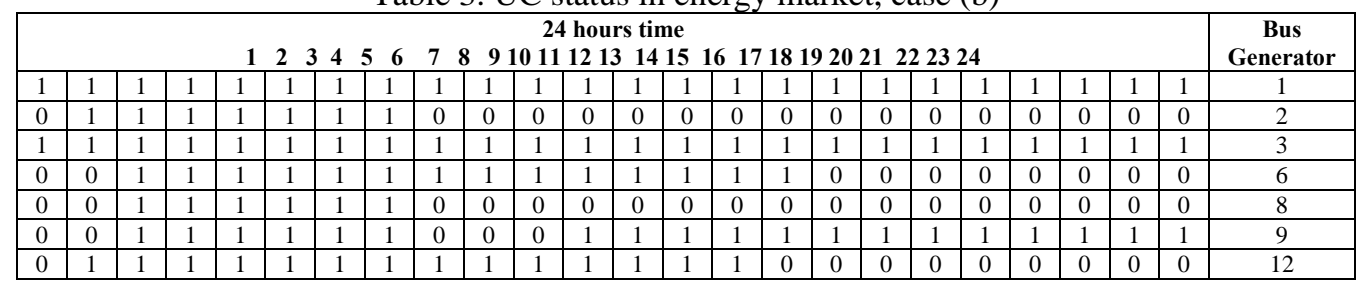

Table 4. UC status in spinning reserve market, case (b)

\begin{tabular}{|c|c|c|c|c|c|c|c|c|c|c|c|c|c|c|c|c|c|c|c|c|c|c|c|c|c|c|c|}
\hline \multicolumn{10}{|c|}{} & \multicolumn{10}{c|}{ Bus } \\
Generator \\
\hline 1
\end{tabular}

Table 5. Switching status, case (b)

\begin{tabular}{|c|c|c|c|c|c|c|c|c|c|c|c|c|c|c|c|c|c|c|c|c|c|c|c|c|c|}
\hline \multicolumn{10}{|c|}{$\mathbf{1 0}$} & \multicolumn{10}{c|}{$\begin{array}{c}\text { switchable } \\
\text { Lines }\end{array}$} \\
\hline 0 & 0 & 1 & 1 & 1 & 1 & 1 & 1 & 0 & 0 & 0 & 0 & 0 & 0 & 0 & 0 & 0 & 0 & 0 & 0 & 0 & 0 & 0 & 1 & $1-15$ \\
\hline 0 & 0 & 1 & 1 & 1 & 1 & 1 & 1 & 0 & 0 & 0 & 0 & 0 & 0 & 0 & 0 & 0 & 0 & 0 & 0 & 0 & 0 & 0 & 1 & $7-29$ \\
\hline 1 & 1 & 0 & 0 & 0 & 0 & 0 & 0 & 1 & 1 & 1 & 1 & 1 & 1 & 1 & 1 & 1 & 1 & 1 & 1 & 1 & 1 & 1 & 1 & $8-9$ \\
\hline 1 & 1 & 1 & 0 & 0 & 0 & 0 & 0 & 1 & 1 & 1 & 1 & 1 & 1 & 1 & 1 & 0 & 0 & 0 & 0 & 0 & 0 & 0 & 1 & $22-38$ \\
\hline 1 & 1 & 0 & 0 & 1 & 1 & 1 & 1 & 0 & 0 & 1 & 1 & 1 & 1 & 1 & 1 & 1 & 1 & 1 & 1 & 1 & 1 & 1 & 0 & $48-49$ \\
\hline 1 & 1 & 1 & 1 & 1 & 1 & 1 & 1 & 0 & 0 & 0 & 0 & 0 & 0 & 0 & 0 & 0 & 0 & 0 & 0 & 0 & 0 & 0 & 0 & $48-44$ \\
\hline 0 & 0 & 1 & 1 & 1 & 1 & 1 & 1 & 0 & 0 & 0 & 0 & 0 & 0 & 0 & 0 & 0 & 1 & 1 & 1 & 1 & 1 & 1 & 1 & $13-15$ \\
\hline 1 & 1 & 0 & 0 & 0 & 0 & 0 & 0 & 1 & 1 & 1 & 1 & 1 & 1 & 1 & 1 & 1 & 0 & 0 & 0 & 0 & 0 & 0 & 1 & $3-4$ \\
\hline 0 & 0 & 0 & 0 & 0 & 0 & 0 & 0 & 1 & 1 & 1 & 1 & 1 & 1 & 1 & 1 & 1 & 1 & 1 & 1 & 1 & 1 & 1 & 1 & $12-16$ \\
\hline 0 & 0 & 0 & 0 & 0 & 0 & 1 & 1 & 1 & 1 & 1 & 1 & 1 & 1 & 1 & 1 & 1 & 0 & 0 & 0 & 0 & 0 & 0 & 0 & $1-2$ \\
\hline
\end{tabular}

Table 6. Operation costs, case (b)

\begin{tabular}{|c|c|c|}
\hline $\begin{array}{c}\text { After switching action without } \\
\text { stability limitation }\end{array}$ & Before switching action & costs \\
\hline $293623 \$$ & $319156 \$$ & Energy market cost \\
\hline $55651 \$$ & $59840 \$$ & Spinning reserve market cost \\
\hline $60042 \$$ & $62544 \$$ & Security network cost \\
\hline
\end{tabular}

- Joint energy and reserve clearing with switching considering the transient stability constraints

Tables 7 and 8 show the UC status of in energy and spinning reserve markets with stability constraints. Considering these constraints, the UC status has been changed. In fact the system costs are higher with stability constraints included. These costs are still lower than the base 
case without switching operations. Table 9 shows the system energy, reserve and security costs. As can be seen these costs decrease by the values of $6.5 \%, 5.5 \%$ and $3 \%$ comparing to the case without switching operations. Table 10 shows the switching status for this case study. As can be seen due to stability constraints opening or closing operations of some switches have performed in different hours comparing to Table 5.

Table 7. UC status in energy market, case (c)

\begin{tabular}{|c|c|c|c|c|c|c|c|c|c|c|c|c|c|c|c|c|c|c|c|c|c|c|c|c|}
\hline \multicolumn{24}{|c|}{24 hours time } & \multirow{2}{*}{$\begin{array}{c}\begin{array}{c}\text { Bus } \\
\text { Generator }\end{array} \\
1\end{array}$} \\
\hline 1 & 1 & 1 & 1 & 1 & 1 & 1 & 1 & 1 & 1 & 1 & 1 & 1 & 1 & 1 & 1 & 1 & 1 & 1 & 1 & 1 & 1 & 1 & 1 & \\
\hline 0 & 1 & 1 & 1 & 1 & 1 & 1 & 1 & 1 & 1 & 0 & 0 & 0 & 0 & 0 & 0 & 0 & 0 & 0 & 0 & 0 & 0 & 0 & 0 & 2 \\
\hline 1 & 1 & 1 & 1 & 1 & 1 & 1 & 1 & 1 & 1 & 1 & 1 & 1 & 1 & 1 & 1 & 1 & 1 & 1 & 1 & 1 & 1 & 1 & 1 & 3 \\
\hline 0 & 1 & 1 & 1 & 1 & 1 & 1 & 1 & 1 & 1 & 1 & 1 & 1 & 1 & 1 & 1 & 1 & 1 & 0 & 0 & 0 & 0 & 0 & 0 & 6 \\
\hline 0 & 0 & 1 & 1 & 1 & 1 & 1 & 1 & 1 & 1 & 1 & 0 & 0 & 0 & 0 & 0 & 0 & 0 & 0 & 0 & 0 & 0 & 0 & 0 & 8 \\
\hline 0 & 1 & 1 & 1 & 1 & 1 & 1 & 1 & 0 & 0 & 0 & 0 & 0 & 0 & 0 & 0 & 0 & 0 & 1 & 1 & 1 & 1 & 1 & 1 & 9 \\
\hline 0 & 1 & 1 & 1 & 1 & 1 & 1 & 1 & 1 & 1 & 1 & 1 & 1 & 1 & 1 & 1 & 1 & 1 & 1 & 1 & 0 & 0 & 0 & 0 & 12 \\
\hline
\end{tabular}

Table 8. UC status in spinning reserve market, case (c)

\begin{tabular}{|c|c|c|c|c|c|c|c|c|c|c|c|c|c|}
\hline & \multicolumn{12}{|c|}{24 hours time } & \multirow{2}{*}{$\begin{array}{c}\begin{array}{c}\text { Bus } \\
\text { Generator }\end{array} \\
1\end{array}$} \\
\hline 1 & 1 & 1 & 1 & 1 & 1 & 1 & 1 & 1 & 1 & 1 & 1 & 1 & \\
\hline 0 & 1 & 1 & 1 & 1 & 1 & 1 & 1 & 1 & 1 & 0 & 0 & 0 & 2 \\
\hline 0 & 0 & 1 & 1 & 1 & 1 & 1 & 1 & 1 & 1 & 1 & 1 & 1 & 3 \\
\hline 0 & 1 & 1 & 1 & 1 & 1 & 1 & 1 & 0 & 0 & 0 & 0 & 0 & 6 \\
\hline 0 & 0 & 0 & 1 & 1 & 1 & 1 & 1 & 1 & 1 & 1 & 0 & 0 & 8 \\
\hline 0 & 1 & 1 & 1 & 1 & 1 & 1 & 1 & 0 & 0 & 0 & 0 & 0 & 9 \\
\hline 0 & 1 & 1 & 1 & 1 & 1 & 1 & 1 & 1 & 1 & 1 & 1 & 1 & 12 \\
\hline
\end{tabular}

Table 9. Operation costs, case (b)

\begin{tabular}{|c|c|c|c|}
\hline $\begin{array}{c}\text { After switching } \\
\text { action with } \\
\text { stability limitation }\end{array}$ & $\begin{array}{c}\text { After switching } \\
\text { action without } \\
\text { stability limitation }\end{array}$ & $\begin{array}{c}\text { Before switching } \\
\text { action }\end{array}$ & costs \\
\hline $298410 \$$ & $293623 \$$ & $319156 \$$ & Energy market cost \\
\hline $56548 \$$ & $55651 \$$ & $59840 \$$ & $\begin{array}{c}\text { Spinning reserve market } \\
\text { cost }\end{array}$ \\
\hline $60667 \$$ & $60042 \$$ & $62544 \$$ & Security network cost \\
\hline
\end{tabular}

Table 10. Switching status, case (c)

\begin{tabular}{|c|c|c|c|c|c|c|c|c|c|c|c|c|c|c|c|c|c|c|c|c|c|c|c|c|}
\hline \multicolumn{24}{|c|}{24 hours time } & \multirow{2}{*}{$\begin{array}{c}\begin{array}{c}\text { switchable } \\
\text { Lines }\end{array} \\
1-15\end{array}$} \\
\hline 0 & 1 & 1 & 1 & 1 & 1 & 1 & 1 & 1 & 1 & 1 & 0 & 0 & 0 & 0 & 0 & 0 & 0 & 0 & 0 & 0 & 0 & 0 & 1 & \\
\hline 0 & 1 & 1 & 1 & 1 & 1 & 1 & 1 & 1 & 1 & 1 & 1 & 1 & 1 & 1 & 1 & 1 & 1 & 1 & 1 & 0 & 0 & 0 & 1 & $7-29$ \\
\hline 1 & 0 & 0 & 0 & 0 & 0 & 0 & 0 & 0 & 0 & 1 & 1 & 1 & 1 & 1 & 1 & 1 & 1 & 1 & 1 & 1 & 1 & 1 & 1 & $8-9$ \\
\hline 1 & 1 & 1 & 0 & 0 & 0 & 0 & 0 & 1 & 1 & 1 & 1 & 1 & 1 & 1 & 1 & 0 & 0 & 0 & 0 & 0 & 0 & 0 & 1 & $22-38$ \\
\hline 1 & 1 & 0 & 0 & 1 & 1 & 1 & 1 & 0 & 0 & 1 & 1 & 1 & 1 & 1 & 1 & 1 & 1 & 1 & 1 & 1 & 1 & 1 & 0 & $48-49$ \\
\hline 1 & 1 & 1 & 1 & 1 & 1 & 1 & 1 & 0 & 0 & 0 & 0 & 0 & 0 & 0 & 0 & 0 & 0 & 0 & 0 & 0 & 0 & 0 & 0 & $48-44$ \\
\hline 0 & 0 & 1 & 1 & 1 & 1 & 1 & 1 & 0 & 0 & 0 & 0 & 0 & 0 & 0 & 0 & 0 & 1 & 1 & 1 & 1 & 1 & 1 & 1 & $13-15$ \\
\hline 1 & 0 & 0 & 0 & 0 & 0 & 1 & 1 & 1 & 1 & 1 & 1 & 1 & 1 & 1 & 1 & 1 & 1 & 1 & 1 & 0 & 0 & 0 & 1 & 3-4 \\
\hline 1 & 0 & 0 & 0 & 0 & 0 & 1 & 1 & 1 & 1 & 1 & 1 & 1 & 1 & 1 & 1 & 1 & 1 & 1 & 1 & 1 & 1 & 1 & 1 & $12-16$ \\
\hline 0 & 0 & 0 & 1 & 1 & 1 & 1 & 1 & 1 & 1 & 1 & 1 & 1 & 1 & 1 & 1 & 1 & 1 & 1 & 1 & 0 & 0 & 0 & 0 & $1-2$ \\
\hline
\end{tabular}

The results show that though the transmission switching is useful for system cost reduction, it may cause transient instability in some instances. Therefore, considering the stability constraints in switching is inevitable. With the stability constraints considered in optimization, the performance of the cost reduction is lower. As can be seen the energy cost reduction considering and neglecting the stability constraints are 6.5 and 8 percent. However, these constraints reduce the risk of instability and reduce the instability costs. 
In joint energy and spinning reserve market clearing with switching operations and neglecting the stability constraints (case (b)), the status of line 15-1 has changed from open to close. Here, this switching is modeled in PSAT. Figure 3 shows that this switching cause instability of unit connected to bus 1 . This shows that it is necessary to consider the stability constraints in the model.

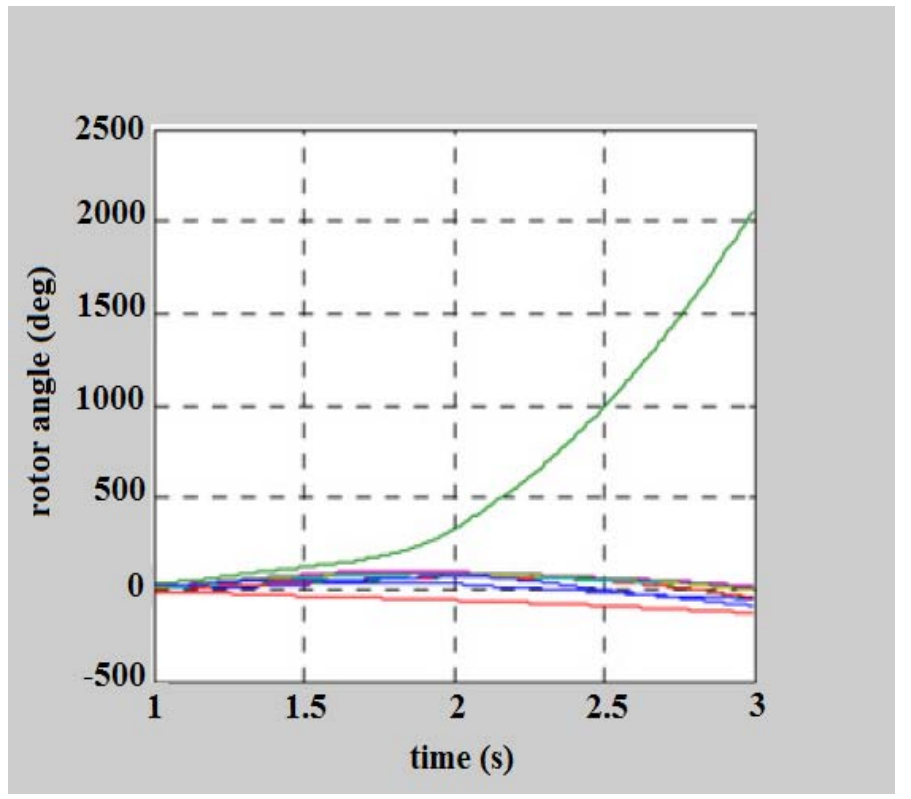

Figure 3. Instability of the unit connected to bus 1 in switching of line 15-1

With stability constraints included, it can be seen that the switching of line 15-1 is performed in hour 13 to14 instead of 16 to 17. This switching does not cause any instability. With stability constraints considered in the model, figureure 4 show the rotor angle of the unit connected to bus 1 during the solution process with the time step of 0.1 second. As can be seen during this switching in time step of 6 the rotor angle reaches the maximum value but in the next step the rotor angle decreases and finally the stability is preserved.

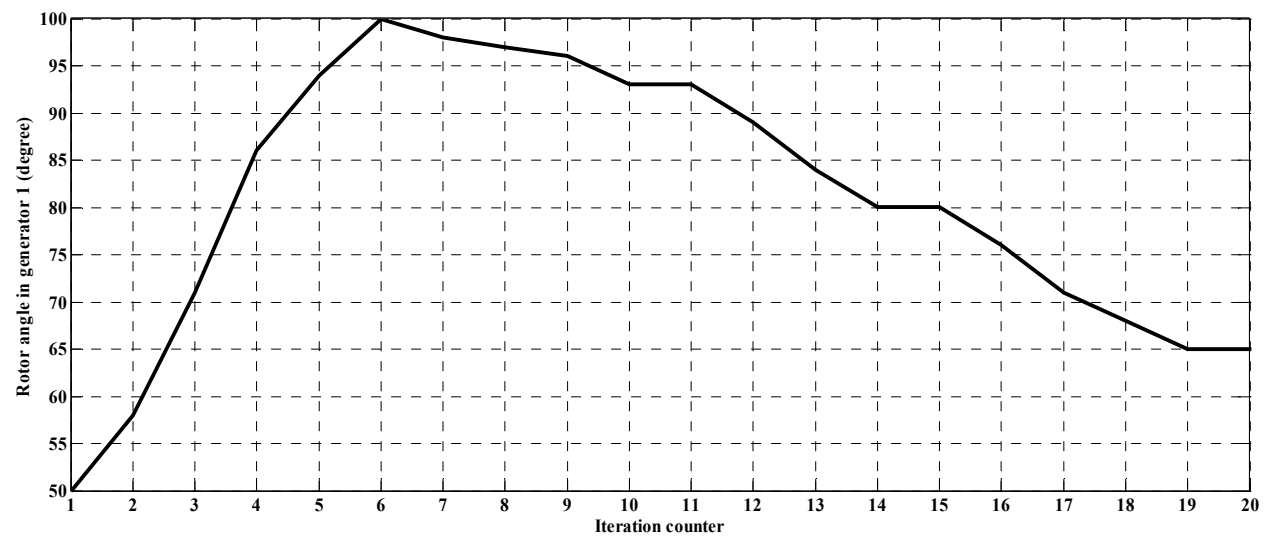

Figure 4- rotor angle of the unit connected to bus 1, with change in the status of line 15-1 For switching of the line 16-12, in case (b) the status of this line is changed from close in hour 16 to open in hour 17. The simulation of this switching in PSAT shows that this switching causes system instability. However, with stability constraints considered (case (c)) this 
switching takes place from hour 18 to hour 19 . The simulations shows that the system is stable in this case.

Figure 5 shows the average marginal price in energy and reserve markets for cases (a), (b) and (c) in 24 hour. As can be seen, these prices are higher for the case including the stability constraints. This increase in the prices with respect to the prices in case study (b) shows that though considering the switching operations in the joint energy and reserve market neglecting the stability constraints leads to the lower prices, this causes the instability in some system units and imposes the high instability cost to the system.

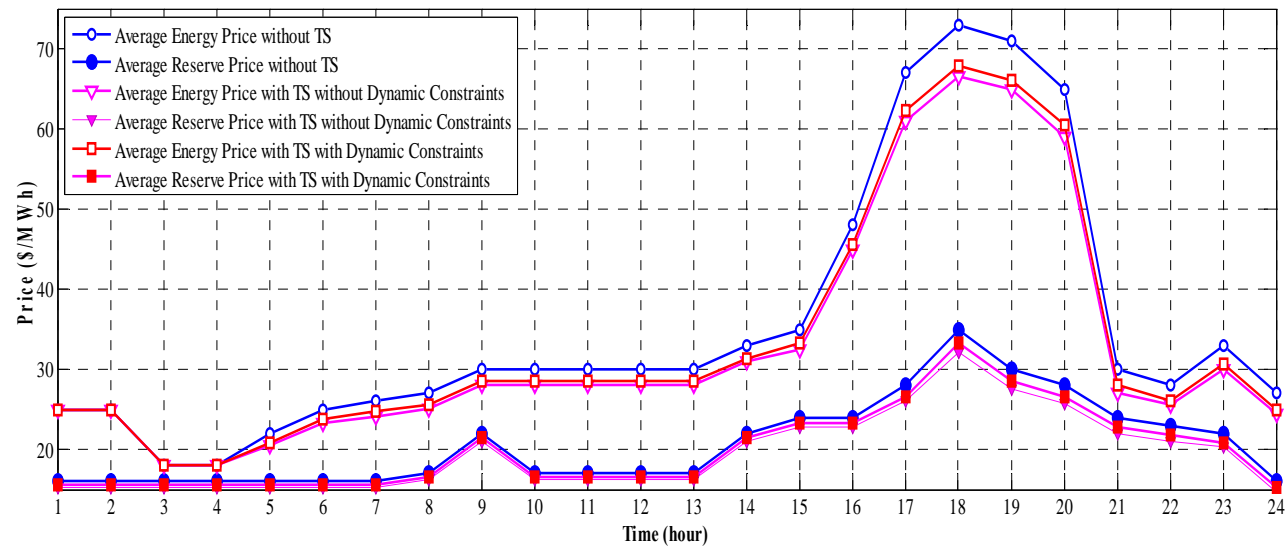

Figure 5. Average marginal price in energy and reserve markets for cases (a), (b) and (c)

The change in Location Marginal Price (LMP) at bus 25 with respect to the change in the number of switchable lines for cases (b) and (c) is shown in figure 6. As can be seen as the number of switchable lines increases the LMP decreases at bus 25. However, this decrease is lower for the case considering the stability constraints.

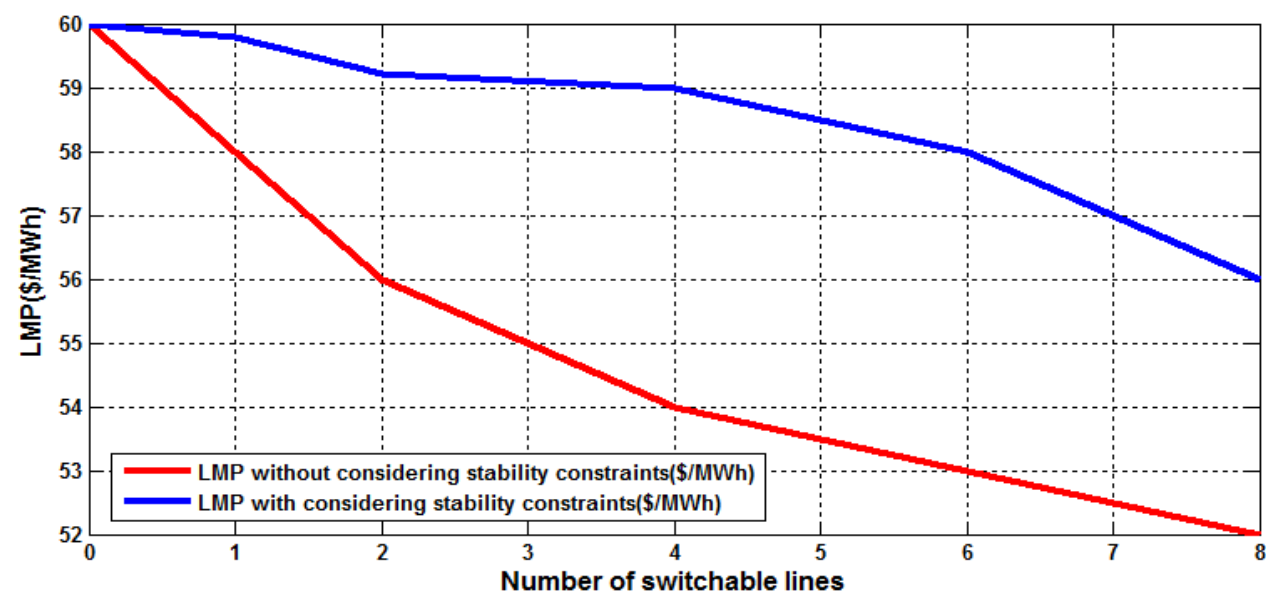

Figure 6. Change in LMP at bus 25 with respect to the change in the number of switchable lines for cases (b) and (c)

Figure 7 shows the change in Expected Energy Not Supplied (EENS) with respect to the change in the number of switchable lines for cases (b) and (c). As can be seen the EENS is higher when the stability constraints are included in the model. With higher number of switchable lines the EENS is lower. 


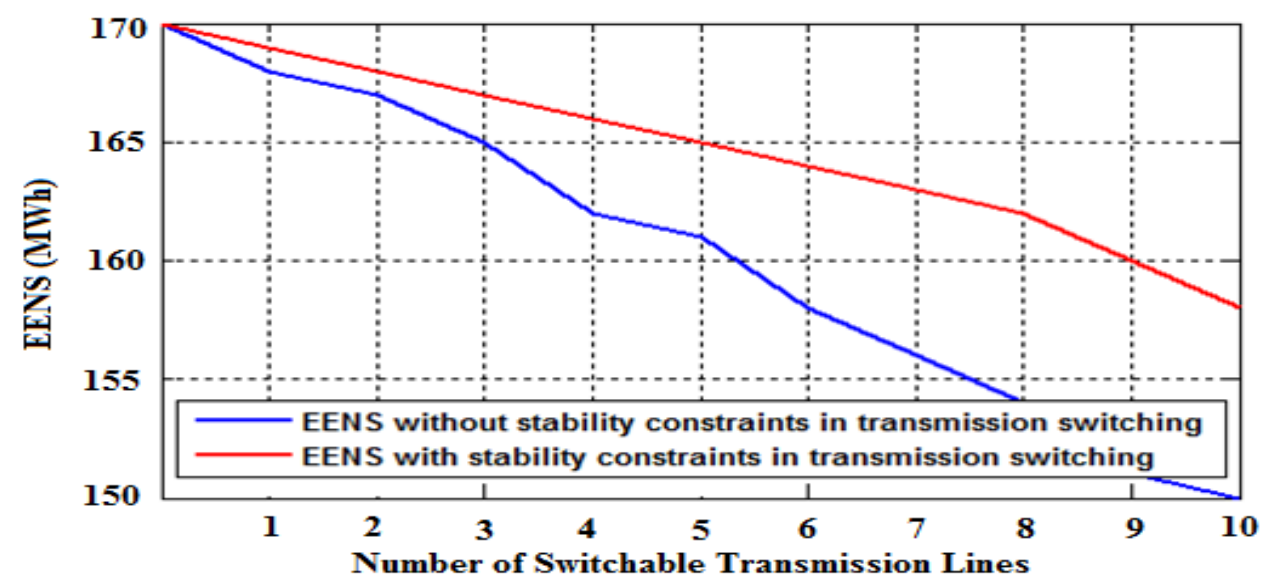

Figure 7. Change in expected energy not supplied with respect to the change in the number of switchable lines for cases (b) and (c)

In addition to reduction of the energy and spinning reserve, transmission switching can improve the voltage at different system buses. Figure 8 shows the Switching effects on voltage at bus 18. The switching has improved the voltage at bus 18 in most of hours. Without switching this voltage is out of allowable range in some instances.

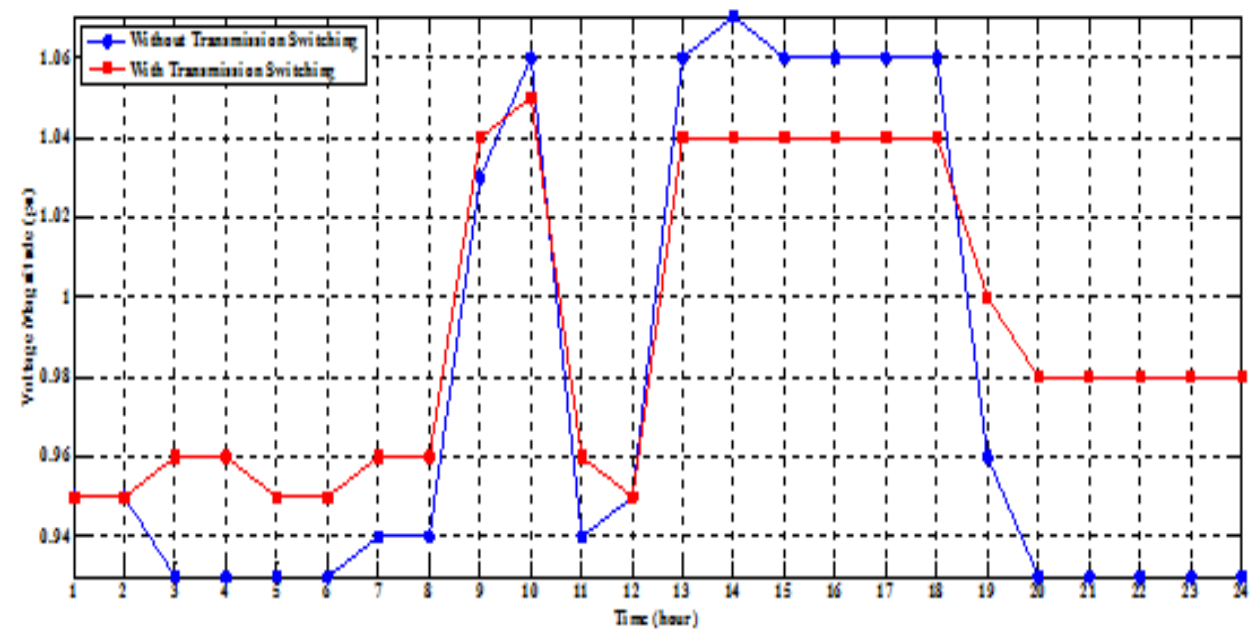

Figure 8. Switching effects on voltage at bus 18

\section{Conclusion}

In this paper the transmission switching has been modeled in joint energy and reserve market clearing. It was observed that through the proper switching operations not only the energy cost but also the spinning reserve and security costs have been reduced. In addition it was shown in case studies that though the switching operation can reduce the operation cost, it may cause dynamic instability and therefore, can impose the additional costs to the system. Therefore, it is necessary to develop appropriate switching strategy to reduce the chance of instability and damage to the units. This paper proposed a methodology to develop such strategies in a day-ahead market. As the results show that the reduction in system cost is lower when the dynamic stability constraints are considered in the mode, but the system stability is preserved under this setup. 


\section{References}

[1] H. J. Koglin and H. Muller, "Overload reduction through corrective switching actions," IEE International Conference on Power System Monitoring and Control, London, pp. 159-164, 1980.

[2] R. V. Amerongen and H. V. Meeteren, "Security control by real power rescheduling network switching and Load shedding," CIGRE Report 32-02, France, 1980.

[3] V. H. Quintana and N. Miiller, "Overload and voltage control of power systems by line switching and generation rescheduling” Canadian Journal Elect. \& Comp. Eng., vol. 15, no.4, pp. 167-173, 1990.

[4] G. Schnyder, H. Glavitsch, "Security enhancement using an optimal switching power flow,” IEEE Trans. on Power Syst., vol. 5, no. 2, pp. 674-681, May 1990.

[5] H. Glavitsch, H. Kronig and R. Bacher, "Combined use of linear programming and load flow techniques in determining optimal switching sequences," Power Systems and Computation Conference (PSCC), Denmark, pp. 627-637, 1984.

[6] W. Shao and V. Vittal, "Corrective switching algorithm for relieving overloads and voltage violations,” IEEE Trans. Power Syst., vol. 20, no. 4, pp. 1877-1885, Nov. 2005.

[7] M. Li, Q. Luh and L. Miche, "Corrective line switching with security constraints for the base and contingency cases," IEEE Trans. Power Syst., vol. 27, no. 1, pp. 125-133, Feb. 2012.

[8] E. B. Fisher, R. P. O’Neill, and M. C. Ferris, “Optimal transmission switching,” IEEE Trans. Power Syst., vol. 23, no. 3, pp. 1364-1355, Aug. 2008.

[9] K. W. Hedman, R. P. O’Neill, E. B. Fisher, and S. S. Oren, "Optimal transmission switching—sensitivity analysis and extensions," IEEE Trans. Power Syst., vol. 23, no. 3, pp. 1469-1479, Aug. 2008.

[10] K. W. Hedman, R. P. O’Neill, E. B. Fisher, and S. S. Oren, "Optimal transmission switching with contingency analysis,” IEEE Trans. Power Syst., vol. 24, no. 3, pp. 15771586, Aug. 2009.

[11] K. W. Hedman, R. P. O'Neill, E. B. Fisher, and S. S. Oren, "Co-Optimization of Generation Unit Commitment and Transmission Switching With N-1 Reliability,” IEEE Trans. Power Syst., vol. 25, no. 2, pp. 1052-1063, May. 2010.

[12] J. D. Fuller, R. Ramasra and A. Chai, "Fast heuristics for transmission line switching," IEEE Trans. Power Syst., vol. 27, no. 3, pp. 1377-1386, Aug. 2012.

[13] C. Liu, J. Wang and J. Ostrowski, "Heuristic prescreening switchable branches in optimal transmission switching," IEEE Trans. Power Syst., vol. 27, no. 4, pp. 2289-2290, Nov. 2012.

[14] P. A. Ruiz, J. M. Foster, A. Rudkevich and M. C. Caramanis, "Tractable transmission topology control using sensitivity analysis," IEEE Trans. Power Syst., vol. 27, no. 3, pp. 1550-1559, Aug. 2012.

[15] M. Khanabadi and H. Ghasemi; "Transmission congestion management through optimal transmission switching”; Power and Energy Society General Meeting, May 2011.

[16] E. Nasrolahpour, H. Ghasemi and M. khanabadi, "Optimal transmission congestion management by means of substation reconfiguration," in Iranian Conference Electrical Engineering (ICEE), 2012.

[17] M. Khanabadi, H. Ghasemi and M. Doostizadeh, "Optimal transmission switching considering voltage security and $\mathrm{N}-1$ contingency analysis," IEEE Trans. Power Syst., vol. 28, no. 1, pp. 542-550, Jan. 2013.

[18] Affonso CM. Silva LCPd. Potential benefits of implementing load management to improve power system security. Int J Electr Power Energy Syst 2010; 32:704-10.

[19] Fu X, Wang X. Determination of load shedding to provide voltage stability. Int J Electr Power Energy Syst 2011; 33:515-21.

[20] L. Wu, M. Shahidehpour, and T. Li, "Stochastic security-constrained unit commitment," IEEE Trans. Power Syst., vol. 22, no. 2, pp. 800-811, May 2007. 
[21] Y. Fu, M. Shahidehpour, and Z. Li, "Security-constrained unit commitment with AC constraints,” IEEE Trans. Power Syst., vol. 20, no. 3, pp. 1538-1550, Aug. 2005.

[22] F. Bouffard, F. D. Galiana, and A. J. Conejo, "Market-clearing with stochastic securityPart I: Formulation,” IEEE Trans. Power Syst., vol. 20, no. 4, pp. 1818-1826, Nov. 2005.

[23] C. Corchero, F. Javier Heredia, "Two-stage Stochastic Programming Model for the Thermal Optimal Day-Ahead Bid Problem with Physical Future Contracts ," IEEE Heredia, Corchero - DR 2008/11 - EIO, UPC. Copies of this report may be downloaded at http://www-eio.upc.es/ corchero/

[24] Q. P. Zheng, J. Wang Panos, M. Pardalos, Y. Guan, "Stochastic Security Constrained Unit Commitment Models and Solution Algorithm", (www2.cemr.wvu.edu/ zheng/paper/ebd4scuc.pdf)

[25] C. C. CARøE, R. SCHULTZ, “A Two-Stage Stochastic Program for Unit Commitment under Uncertainty in a Hydro-Thermal Power System“, Konrad-Zuse-Zentrum fur Information stechnik Berlin Feb 1998.

[26] TAKRITI, S, B. KRASENBRINK, L. S.Y. WU, "Incorporating fuel constraints and electricity spot prices into the stochastic unit commitment problem”, IBM Research Report RC 21066, Yorktown Heights, New York, 1997.

[27] K. Saenchai, L. Benedicenti and G. H. Huang, "A Mixed-Integer two-stage Interval Stochastic Programming Model for Regional Air Quality Management "Environmental Informatics Archives, vol 5, 2007, pp. 168- 176.

[28] S. J. Stoyan, R. H. Kwon, "A two-stage stochastic mixed-integer programming approach to the index tracking problem “ Optim Eng Journal, pp. 247- 275, DOI 10.1007/s11081009-9095-1.

[29] M. Shahidehpour and Y. Fu, "Benders decomposition," IEEE Power and Energy Mag., vol. 3, no. 2, pp. 20-21, Mar. 2005.

[30] S. S. Nielsen, S. A. Zenios,” Scalable parallel Benders decomposition for stochastic linear programming”, Parallel Computing journal, vol. 23, 1997, pp. 1069-1088.

[31] N, Hazrati, M. Rashidi-Nejad, A.A. Gharaveisi, "Pricing and Allocation of Spinning Reserve and Energy in Restructured Power Systems via Memetic Algorithm” Large Engineering Systems Conference on Power Engineering, pp. 234-238, 2007. 